\title{
Autologous Expanded Mesenchymal Stem Cell Implantation for Orthopedics Conditions as a Non- Surgical Approach
}

\author{
Hassan Mubark* \\ Rheumatologist Institution: Auckland Regenerative Clinic, Ormiston Specialist Centre, New Zealand \\ *Corresponding author: Hassan Mubark, Rheumatologist Institution: Auckland Regenerative Clinic, Ormiston Specialist \\ Centre, New Zealand
}

\begin{tabular}{|c|c|}
\hline ARTICLE INFO & ABSTRACT \\
\hline Received: 幽 October 01, 2021 & $\begin{array}{l}\text { Citation: Hassan Mubark. Autologous Expanded Mesenchymal Stem Cell Implantation } \\
\text { for Orthopedics Conditions as a Non-Surgical Approach. Biomed J Sci \& Tech Res 39(2)- }\end{array}$ \\
\hline Published: 豐 October 07, 2021 & 2021. BJSTR. MS.ID.006277. \\
\hline
\end{tabular}

\section{Introduction}

This article aims to address best practices in regenerative medicine using autologous expanded mesenchymal stem cells (MSCs) combined with platelet-rich plasma (PRP). We summarize our findings from various literature reviews and our experience using combined fat-derived expanded MSCs-PRP therapy in many hundreds of clients for osteoarthritis (OA) and tendinopathy, namely tendon tear(s). Generally, the doses we use in large areas ranging between 50-100 million MSCs in 2 MLS of fluid combined with 5-8 MLS of PRP in the same syringe depending on the size of the area(s), but in smaller joints, we modify the number of cells and PRP volume. We also found that concentrated MSCs without PRP is more effective for small joints of the hands and feet. The number of MSCs used in the expanded technique makes the therapy more effective than the low number used in the same-day procedure, i.e., the stromal vascular fraction (SVF); thus, expansion of MSCs is encouraged if the regulatory authority allows this practice. Additionally, we can adjust the volume accordingly by adding more PRP to the mixture to cover more expansive areas. There are three main questions to ask; what is the mechanism of action of this combined therapy? Does it work? And is it safe?

MSCs are multipotent cells with a regenerative effect via both direct and indirect effects. The explicit incorporation into injured and adjacent tissue (i.e., cellular engraftment), while the indirect pathway is related to un-engrafted MSCs producing paracrine signals cytokines that contribute to tissue regeneration by promoting growth and differentiation of local cells [1]. They have an anti-inflammatory effect, immune modulation, and anti-apoptosis (programmed cell death) effect. In addition, MSCs can self-renew and differentiate into various cells like chondrocytes, tenocytes, or other cells depending on where we implant them [2]. PRP works by the activation of the platelets by the release of their intracellular cytoplasmic granules. Those particles contain inflammatory and growth factors which could be responsible for the healing process. The common ones are Platelet-derived growth factor (PDGF), vascular endothelial growth factor (VEGF), transforming growth factor (TGF)-beta, fibroblast growth factor (FGF), and epidermal growth factor (EGF) [3]. Randomized and non-randomized clinical trials showed positive outcomes to the MSC therapy, particularly in knee $\mathrm{OA}$ [4]. In our practice, we found a success rate of an average of 70-80\%, with best results for the knee involvement followed by the hip and shoulder $0 A$; the good results are more seen in appropriately selected candidates with osteoarthritis "non-boneon-bone pathology."

We calculated success rate based on symptom control, quality of life improvement, slowing or stopping the radiologic progression, and eliminating the need for surgery at least in the first five years of its use. We might need to repeat the implantation in some cases. This therapy is favored over the surgery by speedy recovery, comfort in performing routine daily activities, quick return to work and sport. We noticed good effect does vary starting from two weeks post-therapy for up to a year to see the maximum benefit. 
The good news with this technique is that we can cryopreserve the MSCs in liquid nitrogen for up to fifteen years; this will allow us to repeat the implantation if needed or implicate it in other joints. While the degenerative process takes many years thus regeneration is also a slow process; therefore, we feel repeating imaging too early will not reveal the appropriate comparison. We have concluded MSC therapy stops or slows the progression of osteoarthritis and, on some occasions, recovers some of the cartilage thickness, but those are inconsistent findings and slow processes [4,5]. The main obstacle with cartilage regeneration is the lack of direct blood supply but instead takes it from the subchondral bone and nutrients from synovial fluid. Modified scaffolding techniques like exosomes and hyaluronic acid (HA) might potentiate the effect of stem cell therapy. Exosomes are micro-RNA particles that enhance cell-cell communication [6]. HA has viscous-supplementation physiologic and anti-inflammatory, anti-oxidative, and immune-regulatory pharmacologic positive effects in OA [7]. We believe any scaffold(s) with anabolic effect including PRP, HA, exosomes, and collagen would add substantial synergistic benefit to the MSC therapy.

In terms of tendons regeneration, we have found that the combined MSCs-PRP therapy is significantly effective in symptom control, substantially improves range of motion, and may eliminate the need for surgery [8-11]; in our practice, the success rate is 80-90\% of the cases. The clinical benefit was noticed even when there was no clear radiological evidence of tendon healing. We have observed MRI healing of rotator cuff tears and Achilles' tendinopathy, although the result was inconsistent. Some factors might affect the Efficacy of MSCs-PRP therapy, including local anaesthesia use inside the joint or tendon injected due to its toxicity to both MSCs and platelets. Alcohol and non-steroidal anti-inflammatory medicine before and after the stem cell implantation can reduce its Efficacy, while the use of turmeric/Boswellia as natural preparation help to reduce the inflammatory response post-implantations of the stem cells. The safety of autologous MSCs is well tested in several clinical trials; it has shown no evidence of rejection given both the blood and the cell-based therapies are derived from the same individual. There is no evidence of developing malignancies in autologous adult MSCs $[12,13]$. We avoid using MSCs-PRP in patients with active cancer or in remission with invasive cancer for at least five years from its onset to prevent overstimulation of the pre-existing cancer cells. Additionally, both PRP and MSCs have some antimicrobial activity $[14,15]$; thus, infection is infrequent, particularly we sterilize the expanded cells with both antibiotics and antifungal solutions. Also, we adapted single-use sterile PRP tubes.

In summary, we believe this is future medicine. However, more work and research are needed to advance and standardize the best protocol by choosing the correct number of MSCs used in each area and the PRP dose and centrifuge technique to ensure the maximum effective and safe doses.

\section{References}

1. Dominici M, Le Blanc K, I Mueller, I Slaper-Cortenbach, F CMarini. et al. (2006) Minimal criteria for defining multipotent mesenchymal stromal cells. The International Society for Cellular Therapy position statement. Cryotherapy 8(4): 315-317.

2. Kolf CM, Cho E, Tuan RS (2007) Mesenchymal stromal cells. Biology of adult mesenchymal stem cells: regulation of niche, self-renewal and differentiation. Arthritis Res Ther 9(1): 204.

3. Xuetao Xie, Changqing Zhang, Rocky S Tuan (2014) Biology of plateletrich plasma and its clinical application in cartilage repair. Arthritis Research \& Therapy 16(1): 204.

4. Hirotaka Iijima, Takuya Isho, Hiroshi Kuroki, Masaki Takahashi, Tomoki Aoyama, et al. (2018) Effectiveness of mesenchymal stem cells for treating patients with knee osteoarthritis: a meta-analysis toward the establishment of effective regenerative rehabilitation. NPJ Regen Med 3: 15.

5. Hassan Mubark (2019) Resolution of Patellofemoral Bone Marrow Oedema and Subchondral Cysts on MRI Post a Single Autologous FatDerived Expanded Mesenchymal Stem Cell Therapy. Issue 05: Advances in Orthopedics and Sport Medicine ISSN; 2641-6859.

6. Chao Han, Xuan Sun, Ling Liu, Haiyang Jiang, Yan Shen, et al. (2016) Exosomes and Their Therapeutic Potentials of Stem Cells. Volume 2016 |Article ID 7653489. Stem Cell International.

7. RD Altman, A. Manjoo, A Fierlinger, F Niazi, M Nicholls et al. (2015) The mechanism of action for hyaluronic acid treatment in the osteoarthritic knee: a systematic review. BMC Musculoskelet Disord 16: 321.

8. Han L, Fang WL, Jin B, S-C Xu, X Zheng, et al. (2019) Enhancement of tendon-bone healing after rotator cuff injuries using combined therapy with mesenchymal stem cells and platelet-rich plasma. Eur Rev Med Pharmocol Sci 23(20): 9075-9084

9. Charles MD, Christian DR, Cole BJ (2018) The role of biologic therapy in rotator cuff tears and repairs. Curr Rev Musculoskelet Med 11(1): 150161.

10. Hassan Mubark (2020) Regeneration of Full-Thickness Supraspinatus Rotator Cuff Tendon Tear After Treatment with Autologous Mesenchymal Stem Cells. Vol. 2 No.1 (2020): Bio Ortho J.

11. Hassan Mubark (2021) Successful Treatment with Autologous Mesenchymal Stem Cells Therapy for Muscle Wasting Post-Surgical Repair of Achilles Tendon: A Case Report. Issue 03: Advances in Orthopedics and Sport Medicine ISSN; 2641-6859.

12. Meredith Harrison-Brown, Corey Scholes, Kholoud Hafsi, Maimuna Marenah, Jinjie Li, et al. (2019) Efficacy and safety of cultureexpanded, mesenchymal stem/stromal cells for the treatment of knee osteoarthritis: a systematic review protocol. J Orthop Surg Res 14: 3.4

13. Centeno CJ, Al-Sayegh H, Freeman, Jay Smith, William D Murrell et al. (2016) A multi-centre analysis of adverse events among two thousand three hundred and seventy-two adult patients undergoing adult autologous stem cell therapy for orthopaedic conditions [published correction appears in Int Orthop. 2018 Jan;42(1):223]. Int Orthop 40(8): 1755-1765.

14. Lorenzo Drago, Monica Bortolin, Christian Vassena, Silvio Taschieri, Massimo Del Fabbro, et al. (2013) Antimicrobial activity of pure plateletrich plasma against microorganisms isolated from oral cavity. BMC Microbiology volume 13: 47.

15. Francisca Alcayaga-Miranda, Jimena Cuenca, Maroun Khoury (2017) Antimicrobial Activity of Mesenchymal Stem Cells: Current Status and New Perspectives of Antimicrobial Peptide-Based Therapies. Front Immunol 8: 339. 
ISSN: 2574-1241

DOI: 10.26717/BJSTR.2021.39.006277

Hassan Mubark. Biomed J Sci \& Tech Res

(c) (9) This work is licensed under Creative

Submission Link: https://biomedres.us/submit-manuscript.php

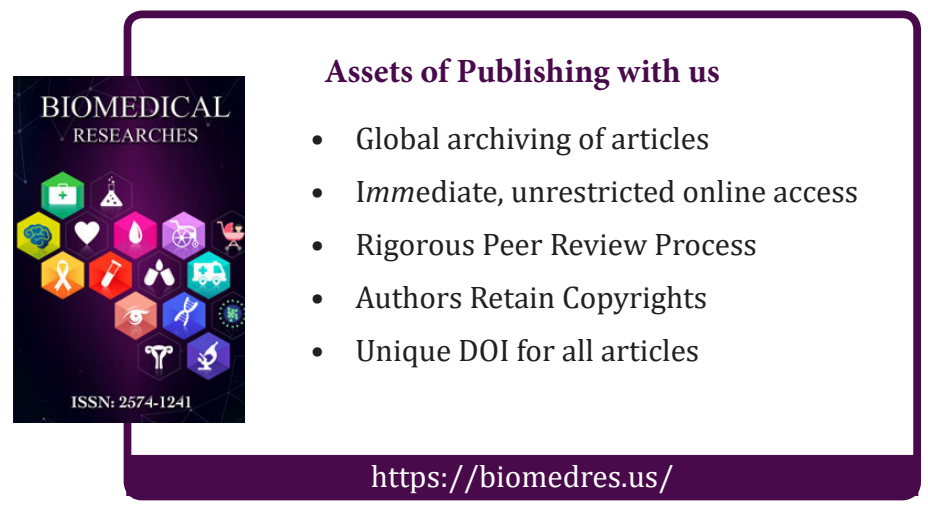

